NSF-KITP-03-82

September 2003

\title{
Prediction of $\mathrm{U}_{\mathrm{e} 3}$
}

\section{in Neutrino Mass Matrix with Two Zeros}

\author{
Mizue Honda *, Satoru Kaneko ${ }^{\dagger}$ \\ and
}

Morimitsu Tanimoto $\ddagger$

Department of Physics, Niigata University, Ikarashi 2-8050, 950-2181 Niigata, JAPAN

\begin{abstract}
We have discussed predictions of $\left|U_{e 3}\right|$ and $J_{C P}$ in the framework of the neutrino mass matrix with two zeros. In the case of the best fit values of $\tan ^{2} \theta_{12}, \tan ^{2} \theta_{23}, \Delta m_{\mathrm{sun}}^{2}$ and $\Delta m_{\mathrm{atm}}^{2}$, the prediction of $\left|U_{e 3}\right|$ is $0.11 \sim 0.14$. The lower bound of $\left|U_{e 3}\right|$ is 0.05 , which depends on $\tan \theta_{12}$ and $\tan \theta_{23}$. We have investigated the stability of these predictions taking account of small corrections to zeros, which may come from radiative corrections or offdiagonal elements of the charged lepton mass matrix. The lower bound of $\left|U_{e 3}\right|$ comes down considerably due to the small corrections to zeros.
\end{abstract}

\footnotetext{
*E-mail address: mizue@muse.sc.niigata-u.ac.jp

$\dagger$ E-mail address: kaneko@muse.sc.niigata-u.ac.jp

${ }^{\ddagger}$ E-mail address: tanimoto@muse.sc.niigata-u.ac.jp
} 


\section{Introduction}

In recent years empirical understanding of the mass and mixing of neutrinos have been advanced $[1,2,3]$. The KamLAND experiment selected the neutrino mixing solution that is responsible for the solar neutrino problem nearly uniquely [4], only large mixing angle solution. We have now good understanding concerning the neutrino mass difference squared $\left(\Delta m_{\mathrm{atm}}^{2}, \Delta m_{\text {sun }}^{2}\right)$ and neutrino flavor mixings $\left(\sin ^{2} 2 \theta_{\mathrm{atm}}, \tan ^{2} \theta_{\text {sun }}\right)$ [5]. A constraint has also been placed on the mixing from the reactor experiment of $\mathrm{CHOOZ} \mathrm{[6].}$

The texture zeros of the neutrino mass matrix have been discussed to explain these neutrino masses and mixings [7, 8, 9]. Recently, Frampton, Glashow and Marfatia [10] found acceptable textures of the neutrino mass matrix with two independent vanishing entries in the basis of the diagonal charged lepton mass matrix. The KamLAND result has stimulated the phenomenological analyses of the texture zeros $[11,12,13,14]$. These results favor texture zeros of the neutrino mass matrix phenomenologically.

However, there are theoretical problems. The first one is the effect of radiative corrections. A specific texture of the lepton mass matrix is not preserved to all orders. For example, non-zero components may evolve in zero-entries of the mass matrix at the low energy scale due to radiative corrections even if the zero texture is realized at the high energy scale. The second one is the choice of the flavor basis. In the model with some flavor symmetry, zeros of the neutrino mass matrix are given while the charged lepton mass matrix has off-diagonal components ${ }^{1}$. Then, zeros of the neutrino mass matrix are polluted after diagonalizing the charged lepton mass matrix. Therefore we need to study the stability of predictions of the texture zeros by taking account of small corrections to zeros.

In this paper, we present detailed study of the neutrino mixing parameter $U_{e 3}$ and CP violating quantity $J_{C P}[18]$, which are expected to be affected by the small corrections to zeros in the neutrino mass matrix. It is found that the predicted $U_{e 3}$ and $J_{C P}$ considerably depend on these corrections.

Predictions of the texture two zeros are presented in section 2. Small corrections to zeros

\footnotetext{
${ }^{1}$ There are some models in which zeros of the neutrino mass matrix are realized in the diagonal basis of the charged lepton mass matrix $[15,16,17]$.
} 
are discussed and the stability of predictions are studied in section 3. Section 4 is devoted to the summary.

\section{Predictions of texture two zeros}

There are 15 textures with two zeros for the effective neutrino mass matrix $M_{\nu}$, which have five independent parameters. The two zero conditions give

$$
\left(M_{\nu}\right)_{a b}=\sum_{i=1}^{3} U_{a i} U_{b i} \lambda_{i}=0, \quad\left(M_{\nu}\right)_{\alpha \beta}=\sum_{i=1}^{3} U_{\alpha i} U_{\beta i} \lambda_{i}=0,
$$

where $\lambda_{i}$ is the $\mathrm{i}$-th eigenvalue including the Majorana phases, and indices $(a b)$ and $(\alpha \beta)$ denote the flavor components, respectively.

Solving these equations, ratios of neutrino masses $m_{1}, m_{2}, m_{3}$, which are absolute values of $\lambda_{i}$ 's, are given in terms of the neutrino mixing matrix $U$ [19] as follows:

$$
\begin{aligned}
& \frac{m_{1}}{m_{3}}=\left|\frac{U_{a 3} U_{b 3} U_{\alpha 2} U_{\beta 2}-U_{a 2} U_{b 2} U_{\alpha 3} U_{\beta 3}}{U_{a 2} U_{b 2} U_{\alpha 1} U_{\beta 1}-U_{a 1} U_{b 1} U_{\alpha 2} U_{\beta 2}}\right|, \\
& \frac{m_{2}}{m_{3}}=\left|\frac{U_{a 1} U_{b 1} U_{\alpha 3} U_{\beta 3}-U_{a 3} U_{b 3} U_{\alpha 1} U_{\beta 1}}{U_{a 2} U_{b 2} U_{\alpha 1} U_{\beta 1}-U_{a 1} U_{b 1} U_{\alpha 2} U_{\beta 2}}\right| .
\end{aligned}
$$

Then, one can test textures in the ratio $R_{\nu}$,

$$
R_{\nu} \equiv\left|\frac{m_{2}^{2}-m_{1}^{2}}{m_{3}^{2}-m_{2}^{2}}\right| \approx \frac{\Delta m_{\mathrm{sun}}^{2}}{\Delta m_{\mathrm{atm}}^{2}}
$$

which has been given by the experimental data. The ratio $R_{\nu}$ is given only in terms of four parameters (three mixing angles and one phase) in

$$
U=\left(\begin{array}{ccc}
c_{13} c_{12} & c_{13} s_{12} & s_{13} e^{-i \delta} \\
-c_{23} s_{12}-s_{23} s_{13} c_{12} e^{i \delta} & c_{23} c_{12}-s_{23} s_{13} s_{12} e^{i \delta} & s_{23} c_{13} \\
s_{23} s_{12}-c_{23} s_{13} c_{12} e^{i \delta} & -s_{23} c_{12}-c_{23} s_{13} s_{12} e^{i \delta} & c_{23} c_{13}
\end{array}\right)
$$

where $c_{i j}$ and $s_{i j}$ denote $\cos \theta_{i j}$ and $\sin \theta_{i j}$, respectively.

Seven acceptable textures with two independent zeros were found for the neutrino mass matrix [10], and they have been studied in detail $[12,13]^{2}$. Among them, the textures $A_{1}$ and $A_{2}$ of ref.[10], which correspond to the hierarchical neutrino mass spectrum, are strongly favored by the recent phenomenological analyses $[11,12,13]$. Therefore, we study these two textures in this paper.

\footnotetext{
${ }^{2}$ Additional two textures may be allowed marginally by current data as shown in ref.[12].
} 
In the texture $A_{1}$, which has two zeros as $\left(M_{\nu}\right)_{e e}=0$ and $\left(M_{\nu}\right)_{e \mu}=0$, the mass ratios are given as

$$
\begin{aligned}
& \frac{m_{1}}{m_{3}}=\left|\frac{s_{13}}{c_{13}^{2}}\left(\frac{s_{12} s_{23}}{c_{12} c_{23}}-s_{13} e^{-i \delta}\right)\right|, \\
& \frac{m_{2}}{m_{3}}=\left|\frac{s_{13}}{c_{13}^{2}}\left(\frac{c_{12} s_{23}}{s_{12} c_{23}}+s_{13} e^{-i \delta}\right)\right| .
\end{aligned}
$$

In the texture $A_{2}$, which has two zeros as $\left(M_{\nu}\right)_{e e}=0$ and $\left(M_{\nu}\right)_{e \tau}=0$, the mass ratios are given as

$$
\begin{aligned}
& \frac{m_{1}}{m_{3}}=\left|\frac{s_{13}}{c_{13}^{2}}\left(\frac{s_{12} c_{23}}{c_{12} s_{23}}+s_{13} e^{-i \delta}\right)\right|, \\
& \frac{m_{2}}{m_{3}}=\left|\frac{s_{13}}{c_{13}^{2}}\left(\frac{c_{12} c_{23}}{s_{12} s_{23}}-s_{13} e^{-i \delta}\right)\right| .
\end{aligned}
$$

It is remarked that the mass ratios of the texture $A_{2}$ are given exactly by replacing $\tan \theta_{23}$ in $A_{1}$ with $-\cot \theta_{23}$.

If $\theta_{12}, \theta_{23}, \theta_{13}$ and $\delta$ are fixed, we can predict $R_{\nu}$ of eq.(3), which is compared with the experimental value $\Delta m_{\text {sun }}^{2} / \Delta m_{\text {atm }}^{2}$. Taking account of the following data with $90 \%$ C.L. [5],

$$
\begin{array}{rlrl}
\sin ^{2} 2 \theta_{\mathrm{atm}} & \geq 0.92, & \Delta m_{\mathrm{atm}}^{2}=(1.5 \sim 3.9) \times 10^{-3} \mathrm{eV}^{2}, \\
\tan ^{2} \theta_{\text {sun }}=0.33 \sim 0.67, & \Delta m_{\mathrm{sun}}^{2}=(6 \sim 8.5) \times 10^{-5} \mathrm{eV}^{2},
\end{array}
$$

with $\sin \theta_{\mathrm{CHOOz}} \leq 0.2$, we predict $R_{\nu}$. In Fig.1, we present the scatter plot of the predicted $R_{\nu}$ versus $\sin \theta_{13}$, in which $\delta$ is taken in the whole range $-\pi \sim \pi$ for the texture $A_{1}$. The parameters are taken in the following ranges of $\theta_{12}=30^{\circ} \sim 39^{\circ}, \theta_{23}=37^{\circ} \sim 53^{\circ}, \theta_{13}=$ $1^{\circ} \sim 12^{\circ}$ and $\delta=-\pi \sim \pi$. It is found that many predicted values of $R_{\nu}$ lie outside the experimental allowed region. This result means that there is a strong constraint for the parameter $\theta_{13}$. We get $\sin \theta_{13} \geq 0.05$ from the experimental value of $R_{\nu}$ as seen in Fig.1.

In order to present the allowed region of $\sin \theta_{13}$ for the texture $A_{1}$, we show the scatter plot of $\sin \theta_{13}$ versus $\tan ^{2} \theta_{12}$ and $\tan ^{2} \theta_{23}$ in Fig.2 and Fig.3, respectively. If we take the best fit values of $\tan ^{2} \theta_{12}=0.42, \tan ^{2} \theta_{23}=1, \Delta m_{\text {sun }}^{2}=7.3 \times 10^{-5} \mathrm{eV}^{2}$ and $\Delta m_{\mathrm{atm}}^{2}=2.5 \times 10^{-3} \mathrm{eV}^{2}$, the prediction of $\sin \theta_{13}$ is $0.11 \sim 0.14$, where the phase $\delta$ is taken in the whole range $-\pi \sim \pi$. In Fig.4, we show $J_{C P}$ versus $\sin \theta_{13}$ for the texture $A_{1}$. Since $J_{C P}$ is proportional to $\sin \theta_{13}$ 
and $\sin \delta$, we also show the allowed region of $\delta$ versus $\sin \theta_{13}$ in Fig.5. It is found that $\delta$ is allowed in the whole range of $-\pi \sim \pi$.

We do not show the numerical results in the texture $A_{2}$ because those are obtained only by replacing $\tan \theta_{23}$ in $A_{1}$ with $-\cot \theta_{23}$.

The allowed regions in Fig.2 and Fig.3 are quantitatively understandable in the following approximate relations:

$$
\left|U_{e 3}\right| \equiv \sin \theta_{13} \simeq \frac{1}{2} \tan 2 \theta_{12} \cot \theta_{23} \sqrt{R_{\nu} \cos 2 \theta_{12}}
$$

for the texture $A_{1}$, and

$$
\left|U_{e 3}\right| \equiv \sin \theta_{13} \simeq \frac{1}{2} \tan 2 \theta_{12} \tan \theta_{23} \sqrt{R_{\nu} \cos 2 \theta_{12}}
$$

for the texture $A_{2}$, respectively, where the phase $\delta$ is neglected because it is a next leading term. As $\tan \theta_{12}$ increases, the lower bound of $\left|U_{e 3}\right|$ increases, and as $\tan \theta_{23}$ decreases, it increases. It is found in Fig.2 that the lower bound $\left|U_{e 3}\right|=0.05$ is given in the case of the smallest $\tan ^{2} \theta_{12}$, while $\left|U_{e 3}\right|=0.08$ is given in the largest $\tan ^{2} \theta_{12}$. On the other hand, as seen in Fig.3, the lower bound $\left|U_{e 3}\right|=0.05$ is given in the largest $\tan ^{2} \theta_{23}$, while $\left|U_{e 3}\right|=0.08$ is given in the smallest $\tan ^{2} \theta_{23}$. The upper bound of $\left|J_{C P}\right|$ is 0.05 , but $J_{C P}=0$ is still allowed in Fig.4. These predicted regions will be reduced in the future since error bars of experimental data in eq.(7) will be reduced, especially, KamLAND is expected to determine $\Delta m_{12}^{2}$ precisely.

\section{$3 \quad$ Stability of predicted $\mathrm{U}_{\mathrm{e} 3}$}

Above predictions are important ones in the texture zeros. The relative magnitude of each entry of the neutrino mass matrix is roughly given as follows:

$$
M_{\nu} \sim\left(\begin{array}{ccc}
0 & 0 & \lambda \\
0 & 1 & 1 \\
\lambda & 1 & 1
\end{array}\right) \quad \text { for } A_{1}, \quad\left(\begin{array}{lll}
0 & \lambda & 0 \\
\lambda & 1 & 1 \\
0 & 1 & 1
\end{array}\right) \quad \text { for } A_{2}
$$

where $\lambda \simeq 0.2$. However, these texture zeros are not preserved to all orders. Even if zero-entries of the mass matrix are given at the high energy scale, non-zero components may evolve instead of zeros at the low energy scale due to radiative corrections. Those magnitudes 
depend on unspecified interactions from which lepton masses are generated. Moreover, zeros of the neutrino mass matrix are given while the charged lepton mass matrix has off-diagonal components in the model with some flavor symmetry. Then, zeros are not realized in the diagonal basis of the charged lepton mass matrix. In other words, zeros of the neutrino mass matrix is polluted by the small off-diagonal elements of the charged lepton mass matrix.

Therefore, one need the careful study of stability of the prediction for $\left|U_{e 3}\right|$ and $J_{C P}$ because these are small quantities. In order to see the effect of the small non-zero components, the conditions of zeros in eq.(1) are modified. The two conditions turn to

$$
\left(M_{\nu}\right)_{a b}=\sum_{i=1}^{3} U_{a i} U_{b i} \lambda_{i}=\epsilon, \quad\left(M_{\nu}\right)_{\alpha \beta}=\sum_{i=1}^{3} U_{\alpha i} U_{\beta i} \lambda_{i}=\omega
$$

where $\epsilon$ and $\omega$ are arbitrary parameters with the mass unit, which are much smaller than other non-zero components of the mass matrix. These parameters are supposed to be real for simplicity. We get

$$
\begin{aligned}
& \frac{m_{1}}{m_{3}}=\left|\frac{U_{13} U_{13} U_{12} U_{22}-U_{12} U_{12} U_{13} U_{23}-U_{12} U_{22} \bar{\epsilon}+U_{12} U_{12} \bar{\omega}}{U_{12} U_{12} U_{11} U_{21}-U_{11} U_{11} U_{12} U_{22}}\right|, \\
& \frac{m_{2}}{m_{3}}=\left|\frac{U_{11} U_{11} U_{13} U_{23}-U_{13} U_{13} U_{11} U_{21}+U_{11} U_{21} \bar{\epsilon}-U_{11} U_{11} \bar{\omega}}{U_{12} U_{12} U_{11} U_{21}-U_{11} U_{11} U_{12} U_{22}}\right|
\end{aligned}
$$

for the texture $A_{1}$, and

$$
\begin{aligned}
& \frac{m_{1}}{m_{3}}=\left|\frac{U_{13} U_{13} U_{12} U_{32}-U_{12} U_{12} U_{13} U_{33}-U_{12} U_{32} \bar{\epsilon}+U_{12} U_{12} \bar{\omega}}{U_{12} U_{12} U_{11} U_{31}-U_{11} U_{11} U_{12} U_{32}}\right| \\
& \frac{m_{2}}{m_{3}}=\left|\frac{U_{11} U_{11} U_{13} U_{33}-U_{13} U_{13} U_{11} U_{31}+U_{11} U_{31} \bar{\epsilon}-U_{11} U_{11} \bar{\omega}}{U_{12} U_{12} U_{11} U_{31}-U_{11} U_{11} U_{12} U_{32}}\right|
\end{aligned}
$$

for the texture $A_{2}$, where $\bar{\epsilon}$ and $\bar{\omega}$ are normalized ones as $\bar{\epsilon}=\epsilon / \lambda_{3}$ and $\bar{\omega}=\omega / \lambda_{3}$, respectively. We obtain approximately

$$
\begin{aligned}
& \frac{m_{1}}{m_{3}} \simeq\left|t_{12} t_{23} s_{13} e^{-i \delta}-\frac{t_{12}}{c_{23}} \bar{\omega}+\bar{\epsilon}\right| \\
& \frac{m_{2}}{m_{3}} \simeq\left|\frac{1}{t_{12}} t_{23} s_{13} e^{-i \delta}-\frac{1}{t_{12} c_{23}} \bar{\omega}-\bar{\epsilon}\right|
\end{aligned}
$$

for the texture $A_{1}$, and

$$
\begin{aligned}
& \frac{m_{1}}{m_{3}} \simeq\left|-t_{12} \frac{1}{t_{23}} s_{13} e^{-i \delta}+\frac{t_{12}}{s_{23}} \bar{\omega}+\bar{\epsilon}\right| \\
& \frac{m_{2}}{m_{3}} \simeq\left|-\frac{1}{t_{12} t_{23}} s_{13} e^{-i \delta}+\frac{1}{t_{12} s_{23}} \bar{\omega}-\bar{\epsilon}\right|
\end{aligned}
$$


for the texture $A_{2}$, where $t_{i j}=\tan \theta_{i j}$. We present the approximate expression of $\left|U_{e 3}\right|=$ $\sin \theta_{13}$ as follows:

$$
\sin \theta_{13} \simeq \pm \frac{1}{2} \tan 2 \theta_{12} \cot \theta_{23} \sqrt{R_{\nu} \cos 2 \theta_{12}}+\frac{\bar{\omega} \cos \delta}{s_{23}}+\frac{t_{12}}{t_{23}} \frac{\bar{\epsilon} \cos \delta}{1-t_{12}^{2}}+O\left(\bar{\omega}^{2}, \bar{\epsilon}^{2}\right)
$$

for the texture $A_{1}$, and

$$
\sin \theta_{13} \simeq \pm \frac{1}{2} \tan 2 \theta_{12} \tan \theta_{23} \sqrt{R_{\nu} \cos 2 \theta_{12}}+\frac{\bar{\omega} \cos \delta}{c_{23}}-t_{12} t_{23} \frac{\bar{\epsilon} \cos \delta}{1-t_{12}^{2}}+O\left(\bar{\omega}^{2}, \bar{\epsilon}^{2}\right)
$$

for the texture $A_{2}$. In these equations, $+(-)$ of the first term in the right-hand side corresponds to the case of $\tan \theta_{12} \tan \theta_{23}>0(<0)$. It is remarked that the second and third terms in the right-hand side could be comparable with the first one in eq.(16) and eq.(17).

However, the second and third terms in the right-hand side could be partially canceled each other depending on the $\operatorname{sign}$ of $\tan \theta_{12}$ and $\tan \theta_{23}$. Therefore, the prediction of $\left|U_{e 3}\right|$ is somewhat different between the texture $A_{1}$ and $A_{2}$.

In order to estimate the effect of $\bar{\omega}$ and $\bar{\epsilon}$, we consider the case in which the charged lepton mass matrix has small off-diagonal components. Suppose that the two zeros in eq.(10) is still preserved for the neutrino sector. The typical model of the charged lepton is the GeorgiJarlskog texture [20], in which the charged lepton mass matrix $M_{E}$ is given as

$$
M_{E} \simeq\left(\begin{array}{ccc}
0 & \sqrt{m_{e} m_{\mu}} & 0 \\
\sqrt{m_{e} m_{\mu}} & m_{\mu} & \sqrt{m_{e} m_{\tau}} \\
0 & \sqrt{m_{e} m_{\tau}} & m_{\tau}
\end{array}\right)
$$

where each matrix element is written in terms of the charged lepton masses, and phases are neglected for simplicity. This matrix is diagonalized by the unitary matrix $U_{E}$, in which the mixing between the first and second families is $\sqrt{\frac{m_{e}}{m_{\mu}}} \simeq 0.07$ and the mixing between the second and third families is $\sqrt{\frac{m_{e}}{m_{\tau}}} \simeq 0.02$. Since the neutrino mass matrix is still the texture $A_{1}$ or the texture $A_{2}{ }^{3}$, it turns to $A_{1}^{\prime}$ or $A_{2}^{\prime}$ as follows:

$$
M_{\nu} \sim\left(\begin{array}{ccc}
\kappa^{2} & \kappa & \lambda \\
\kappa & 1 & 1 \\
\lambda & 1 & 1
\end{array}\right) \quad \text { for } A_{1}^{\prime}, \quad\left(\begin{array}{ccc}
2 \kappa \lambda & \lambda & \kappa \\
\lambda & 1 & 1 \\
\kappa & 1 & 1
\end{array}\right) \quad \text { for } A_{2}^{\prime}
$$

in the diagonal basis of the charged lepton mass matrix. Therefore, the parameter $\bar{\epsilon}$ are correlated with $\bar{\omega}$ such as $\bar{\omega} \simeq \kappa / 2$ and $\bar{\epsilon} \simeq \kappa^{2} / 2$ in the texture $A_{1}^{\prime}$, and $\bar{\omega} \simeq \kappa / 2$ and $\bar{\epsilon} \simeq \kappa \lambda$ in the texture $A_{2}^{\prime}$.

\footnotetext{
${ }^{3}$ The combined model with Georgi-Jarlskog texture and the $A_{2}$ type one is presented in ref.[14]
} 
By using the texture $A_{1}^{\prime}$ of the neutrinos in eq.(19), we show our results of the allowed region of $\sin \theta_{13}$ versus $\tan ^{2} \theta_{12}$ and $\tan ^{2} \theta_{23}$ in Fig.6 and Fig.7, respectively, where $\tan \theta_{12}$ and $\tan \theta_{23}$ are taken to be positive. In the case of the best fit values of $\tan ^{2} \theta_{12}, \tan ^{2} \theta_{23}$, $\Delta m_{\text {sun }}^{2}$ and $\Delta m_{\text {atm }}^{2}$, the prediction of $\sin \theta_{13}$ is $0.075 \sim 0.15$, which is somewhat wider than the result of the section 2 due to the correction $\kappa$. We have also calculated in the case of $\tan \theta_{12} \tan \theta_{23}<0$. Predictions are almost same because of $\bar{\omega} \gg \bar{\epsilon}$. In Fig.8, we show $J_{C P}$ versus $\sin \theta_{13}$. We also show the allowed region of $\delta$ versus $\sin \theta_{13}$ in Fig.9. These results should be compared with the ones in Fig. $2 \sim$ Fig. 5 . It is noticed that the lower bound of $\sin \theta_{13}$ considerably comes down due to the correction $\kappa$. The small $\left|U_{e 3}\right|$ of $8 \times 10^{-3}$ is allowed. In Fig.6 and Fig.7, we also find that there is an upper bound on $\left|U_{e 3}\right|$ for small values of $\tan ^{2} \theta_{12}$ and large values of $\tan ^{2} \theta_{23}$. We have checked numerically that this upper bound appears due to the higher order terms, which are omitted in eqs.(14), (15), (16) and (17). As seen in Fig.9, the phase $\delta$ is not allowed in the whole range $-\pi \sim \pi$ if $\sin \theta_{12} \leq 0.09$.

In the texture $A_{2}^{\prime}$ of eq.(19), we show allowed regions of $\sin \theta_{13}$ versus $\tan ^{2} \theta_{12}$ and $\tan ^{2} \theta_{23}$ in Fig.10 and Fig.11, respectively. In the case of the best fit values of $\tan ^{2} \theta_{12}, \tan ^{2} \theta_{23}, \Delta m_{\text {sun }}^{2}$ and $\Delta m_{\text {atm }}^{2}$, the prediction of $\sin \theta_{13}$ is $0.08 \sim 0.195$, which is somewhat different from the result of $A_{1}^{\prime}$. There is no upper bound on $\left|U_{e 3}\right|$ in Fig.10 and Fig.11. The effect of $\bar{\omega}$ is canceled partially by the effect of $\bar{\epsilon}$ as seen in eq.(17). Therefore, allowed regions in Fig.10 and Fig.11 are somewhat different from ones in Fig.6 and Fig.7. We show the result in the case of $\tan \theta_{12} \tan \theta_{23}<0$ in Fig.12 and Fig.13. The lower bound of $\sin \theta_{13}$ comes down to zero, which is contrasted with the result in Fig.10 and Fig.11 because there is no cancellation between the effect of $\bar{\omega}$ and $\bar{\epsilon}$ as seen in eq.(17). We omit the figure of $J_{C P}$ because it is almost same as the result in the case of $A_{1}^{\prime}$.

This result depends on a specific model. In order to complete the study of the stability, we also consider the case that two corrections $\bar{\epsilon}$ and $\bar{\omega}$ are independent each other, which may be the case if the deviation from zero arises from radiative corrections. For the texture $A_{1}^{\prime}$, we show the allowed region of $\sin \theta_{13}$ versus $2 \bar{\omega}$ in Fig.14, where $\bar{\epsilon}=0$ is taken. The gray region is allowed by the experimental data for the texture $A_{1}^{\prime}$. The allowed region of the texture $A_{2}^{\prime}$ is almost same as the one of $A_{1}^{\prime}$. In Fig.14, the narrow deep gray region is only added in the case of the texture $A_{2}^{\prime}$. We also show the allowed region of $\sin \theta_{13}$ versus 
$\bar{\epsilon}$ in Fig.15, in which $\bar{\omega}=0$ is taken. There is no difference between the allowed region of both $A_{1}^{\prime}$ and $A_{2}^{\prime}$.

The predicted lower bound of $\left|U_{e 3}\right|$ is sensitive to $\bar{\omega}$. If the correction $\bar{\omega}$ is as large as 0.02 , the lower bound of $\left|U_{e 3}\right|$ comes down to 0.025. If $\bar{\omega}$ is larger than 0.04 , the predictability of $\left|U_{e 3}\right|$ is lost since $\left|U_{e 3}\right|=0$ is allowed. On the other hand, the lower bound is rather insensitive to $\bar{\epsilon}$. Even if the correction $\bar{\epsilon}$ grows up to be 0.1 , the lower bound of $\left|U_{e 3}\right|$ is still 0.02. It is concluded that the magnitude of the deviation from the zero in the $(1,2) /(1,3)$ element for the texture $A_{1} / A_{2}$ is important to predict $\left|U_{e 3}\right|$.

\section{Summary}

We summarize our results as follows. We have studied $\left|U_{e 3}\right|$ and $J_{C P}$ in the textures $A_{1}$ and $A_{2}$ of the neutrino mass matrix with two zeros. The lower bound of $\left|U_{e 3}\right|$ is 0.05 , which considerably depends on $\tan ^{2} \theta_{12}$ and $\tan ^{2} \theta_{23}$. We have investigated the stability of these predictions by taking account of small corrections, which may come from radiative corrections or off-diagonal elements of the charged lepton mass matrix. The lower bound of $\left|U_{e 3}\right|$ comes down significantly in the case of $\bar{\omega} \gg 0.01$, while $\bar{\epsilon}$ is rather insensitive to $\left|U_{e 3}\right|$. Therefore the corrections to the texture zeros in the $(1,2) /(1,3)$ element for the texture $A_{1} / A_{2}$ should be carefully taken to predict $\left|U_{e 3}\right|$.

In the case of best fit values of $\tan ^{2} \theta_{12}, \tan ^{2} \theta_{23}, \Delta m_{\text {sun }}^{2}$ and $\Delta m_{\text {atm }}^{2}$, the prediction of $\left|U_{e 3}\right|$ is $0.075 \sim 0.195$ even if the corrections are taken into account. This prediction is good news for the near future experiments. The measurement of $\left|U_{e 3}\right|$ will be an important test of the texture zeros in the future.

We would like to thank M. Katsumata, H. Nakano and H. So for helpful discussions. We are also grateful to KITP in UCSB, where the paper was completed, for its warm hospitality. This research was supported in part by the National Science Foundation in USA under Grant No. PHY99-07949 and the Grant-in-Aid for Science Research, Ministry of Education, Science and Culture, Japan(No.12047220). 


\section{References}

[1] Super-Kamiokande Collaboration, Y. Fukuda et al, Phys. Rev. Lett. 81 (1998) 1562; ibid. 82 (1999) 2644; ibid. 82 (1999) 5194.

[2] Super-Kamiokande Collaboration, S. Fukuda et al. Phys. Rev. Lett. 86, 5651; 5656 (2001).

[3] SNO Collaboration: Q. R. Ahmad et al., Phys. Rev. Lett. 87 (2001) 071301; nucl-ex/0204008, 0204009.

[4] KamLAND Collaboration, K. Eguchi et al., hep-ex/0212021.

[5] G. L. Fogli, E. Lisi, M. Marrone, D. Montanino, A. Palazzo and A.M. Rotunno, hep$\mathrm{ph} / 0212127$;

J. N. Bahcall, M. C. Gonzalez-Garcia and C. Peña-Garay, JHEP 0302 (2003) 009;

M. Maltoni, T. Schwetz and J.W.F. Valle, hep-ph/0212129;

P.C. Holanda and A. Yu. Smirnov, hep-ph/0212270;

V. Barger and D. Marfatia, hep-ph/0212126.

[6] CHOOZ Collaboration, M. Apollonio et al., Phys. Lett. B466 (1999) 415.

[7] H. Nishiura, K. Matsuda and T. Fukuyama, Phys. Rev. D60 (1999) 013006.

[8] E. K. Akhmedov, G. C. Branco, M. N. Rebelo, Phys. Rev. Lett. 84 (2000) 3535.

[9] S.K. Kang and C.S. Kim, Phys. Rev. D63 (2001) 113010.

[10] P.H. Frampton, S.L. Glashow and D. Marfatia, Phys. Lett. B536 (2002) 79.

[11] Z. Xing, Phys. Lett. B530 (2002) 159.

[12] W. Guo and Z. Xing, hep-ph/0212142.

[13] R. Barbieri, T. Hambye and A. Romanino, hep-ph/0302118.

[14] M. Bando and M. Obara, hep-ph/0212242, 0302034. 
[15] A. Zee, Phys. Lett. B93 (1980) 389; B161 (1985) 141;

L. Wolfenstein, Nucl. Phys. B175 (1980) 92;

S. T. Petcov, Phys. Lett. B115 (1982) 401;

C. Jarlskog, M. Matsuda, S. Skadhauge and M. Tanimoto, Phys. Lett. B449 (1999) 240;

P. H. Frampton and S. Glashow, Phys. Lett. B461 (1999) 95.

[16] P.H. Frampton, M.C. Oh and T. Yoshikawa, Phys. Rev. D66 (2002) 033007.

[17] A. Kageyama, S. Kaneko, N. Shimoyama and M. Tanimoto, Phys. Lett. B538 (2002) 96.

[18] C. Jarlskog, Phys. Rev. Lett. 55 (1985) 1039.

[19] Z. Maki, M. Nakagawa and S. Sakata, Prog. Theor. Phys. 28 (1962) 870.

[20] H. Georgi and C. Jarlskog, Phys. Lett. B86 (1979) 297. 


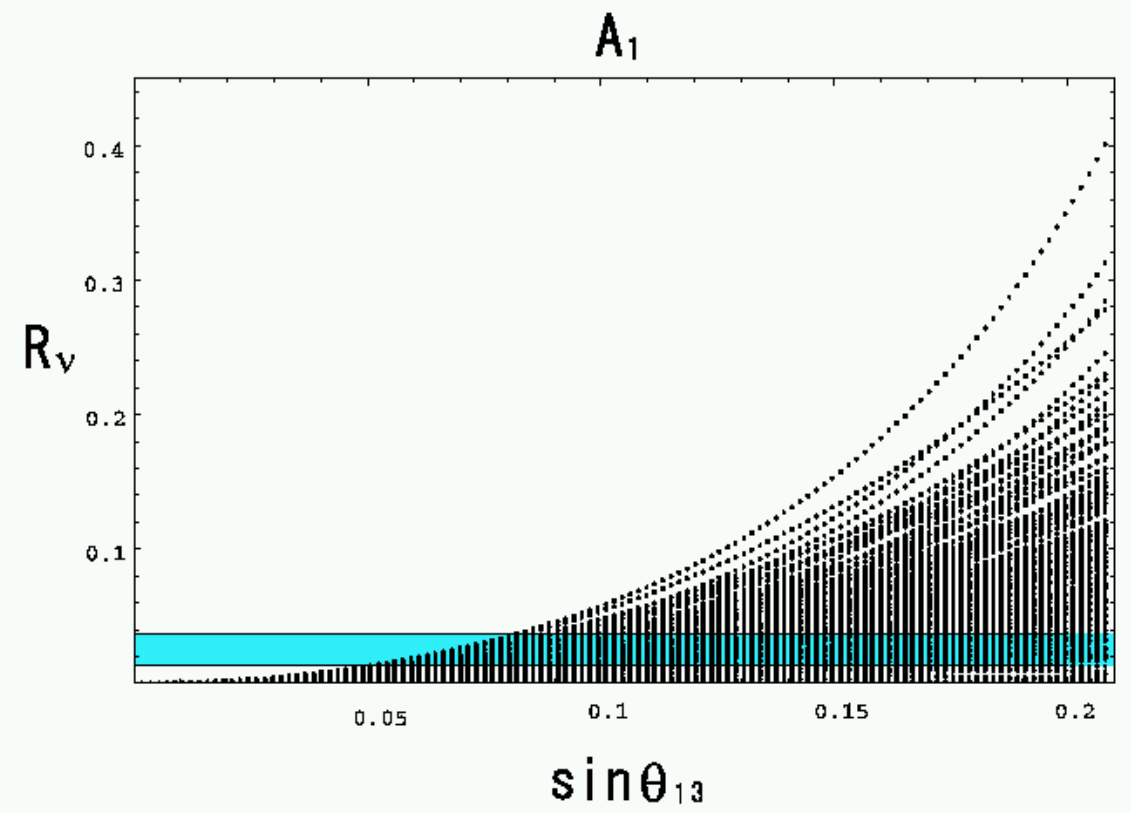

Figure 1: Scatter plot of $R_{\nu}$ versus $\sin \theta_{13}$ for $A_{1}$. The unknown phase $\delta$ is taken in the whole range $-\pi \sim \pi$. The gray horizontal band is the experimental allowed region. 


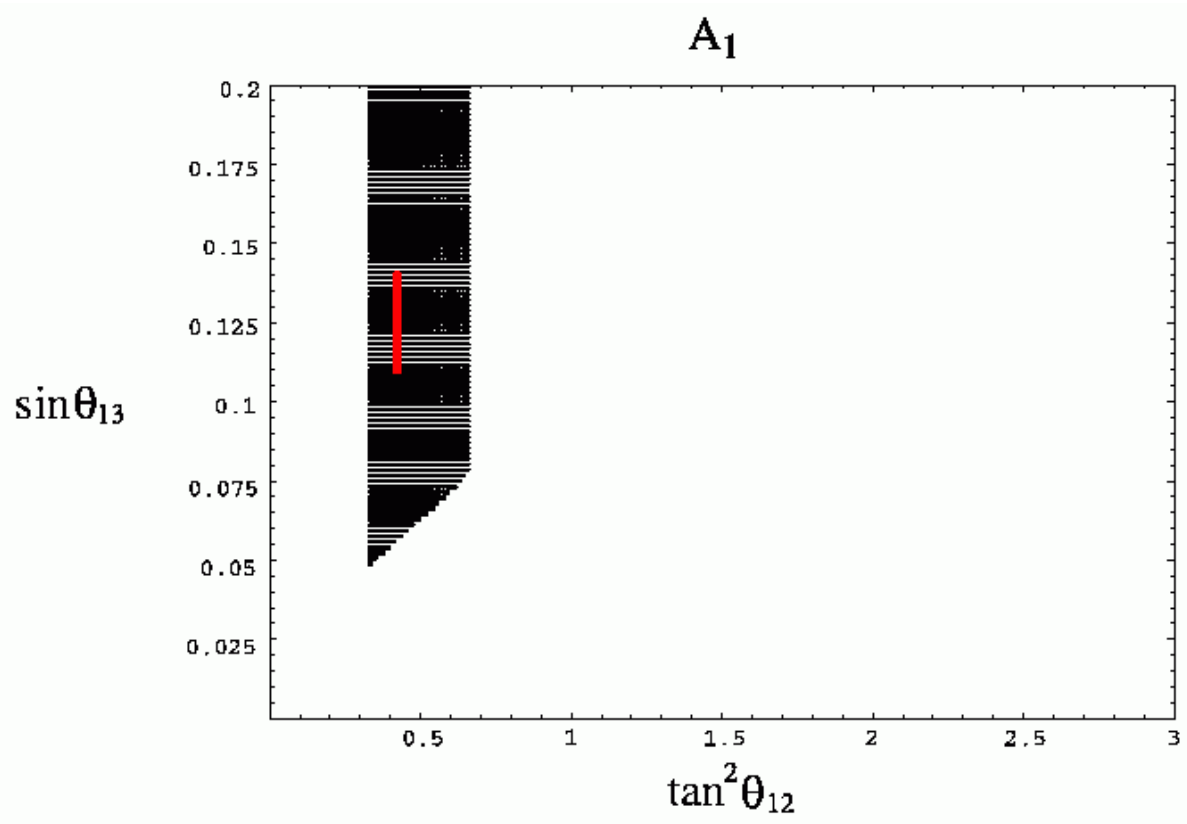

Figure 2: Scatter plot of $\sin \theta_{13}$ versus $\tan ^{2} \theta_{12}$ for $A_{1}$. The best fit is shown by a red line.

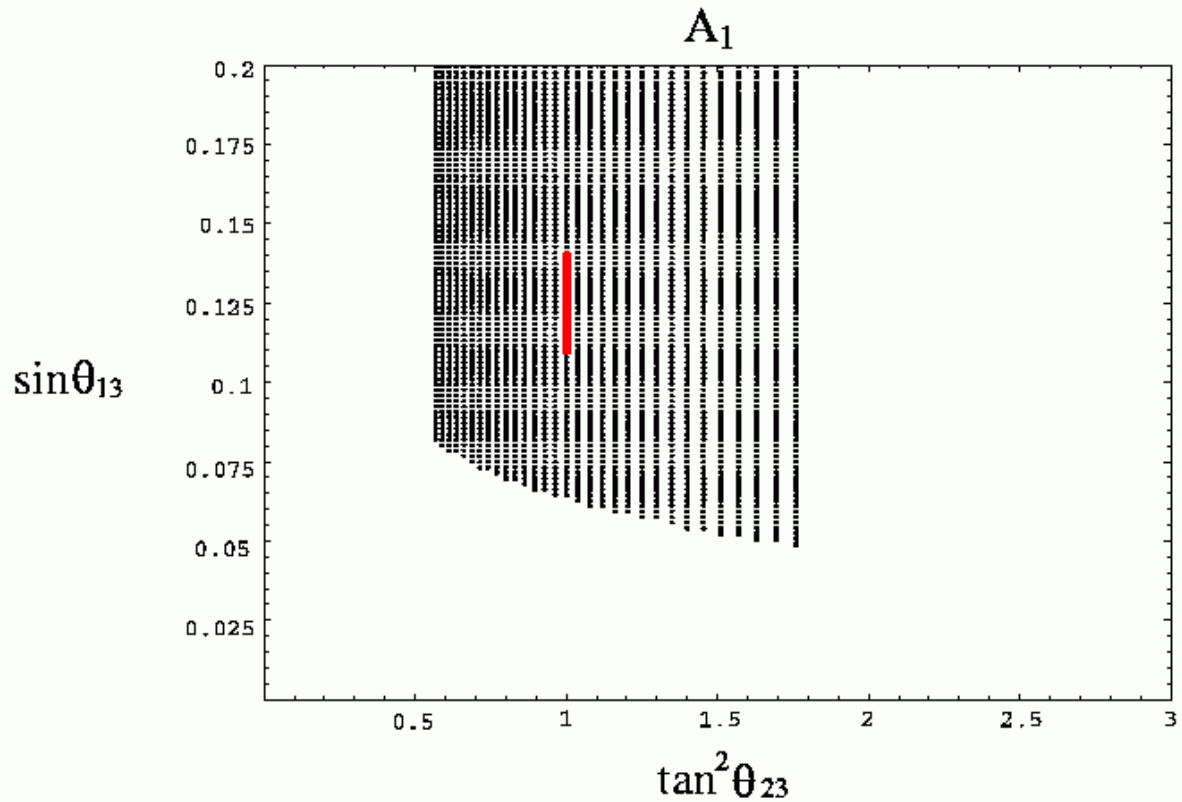

Figure 3: Scatter plot of $\sin \theta_{13}$ versus $\tan ^{2} \theta_{23}$ for $A_{1}$. The best fit is shown by a red line. 


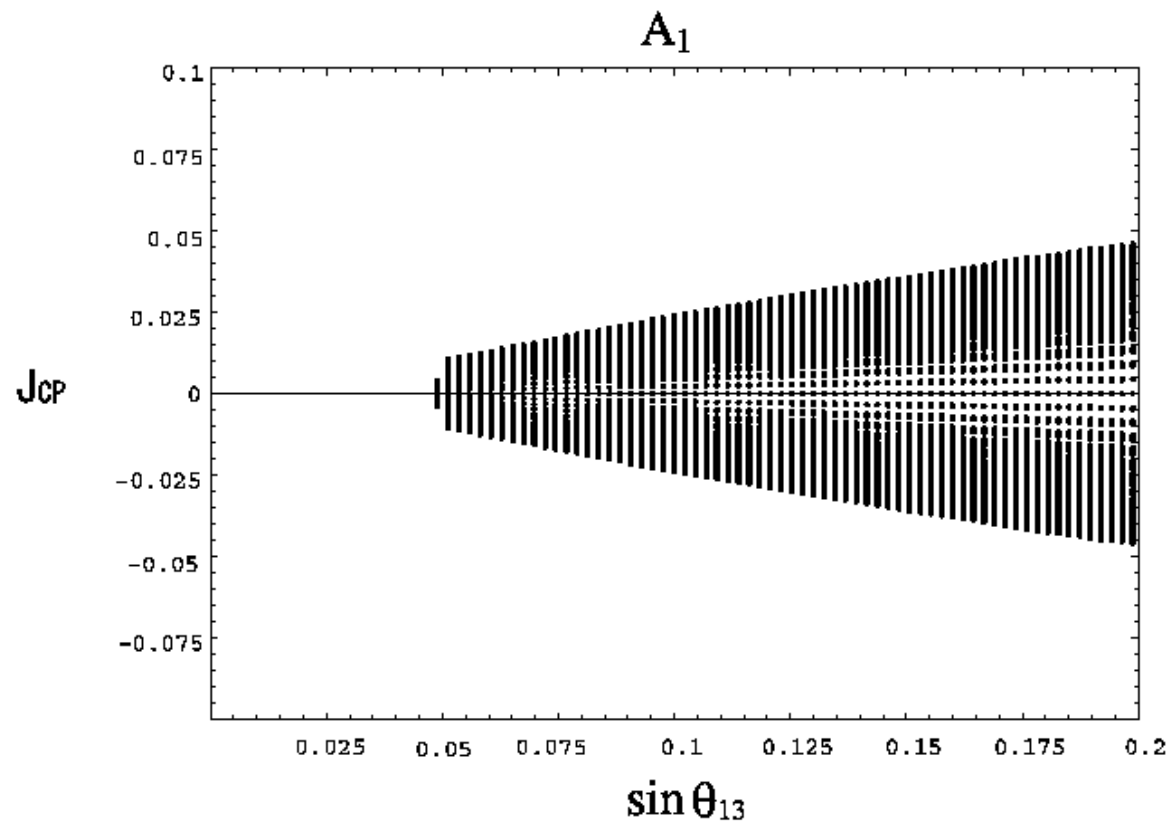

Figure 4: Scatter plot of $J_{C P}$ versus $\sin \theta_{13}$ for $A_{1}$.

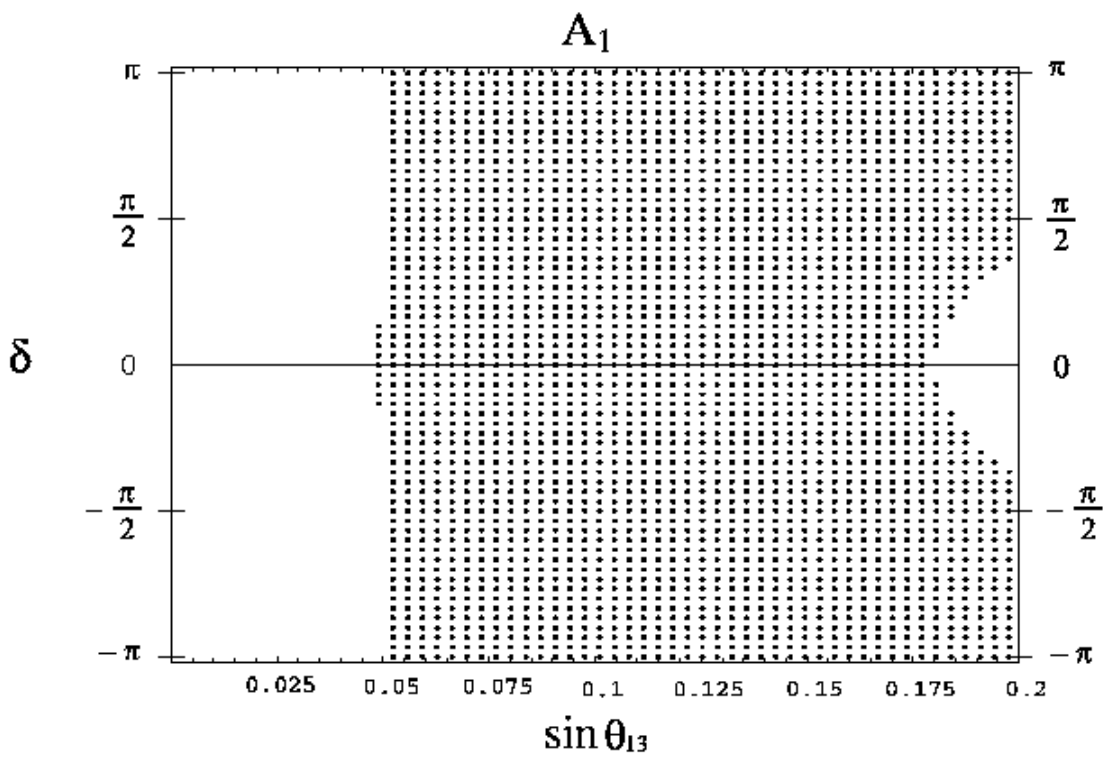

Figure 5: Scatter plot of $\delta$ versus $\sin \theta_{13}$ for $A_{1}$. 


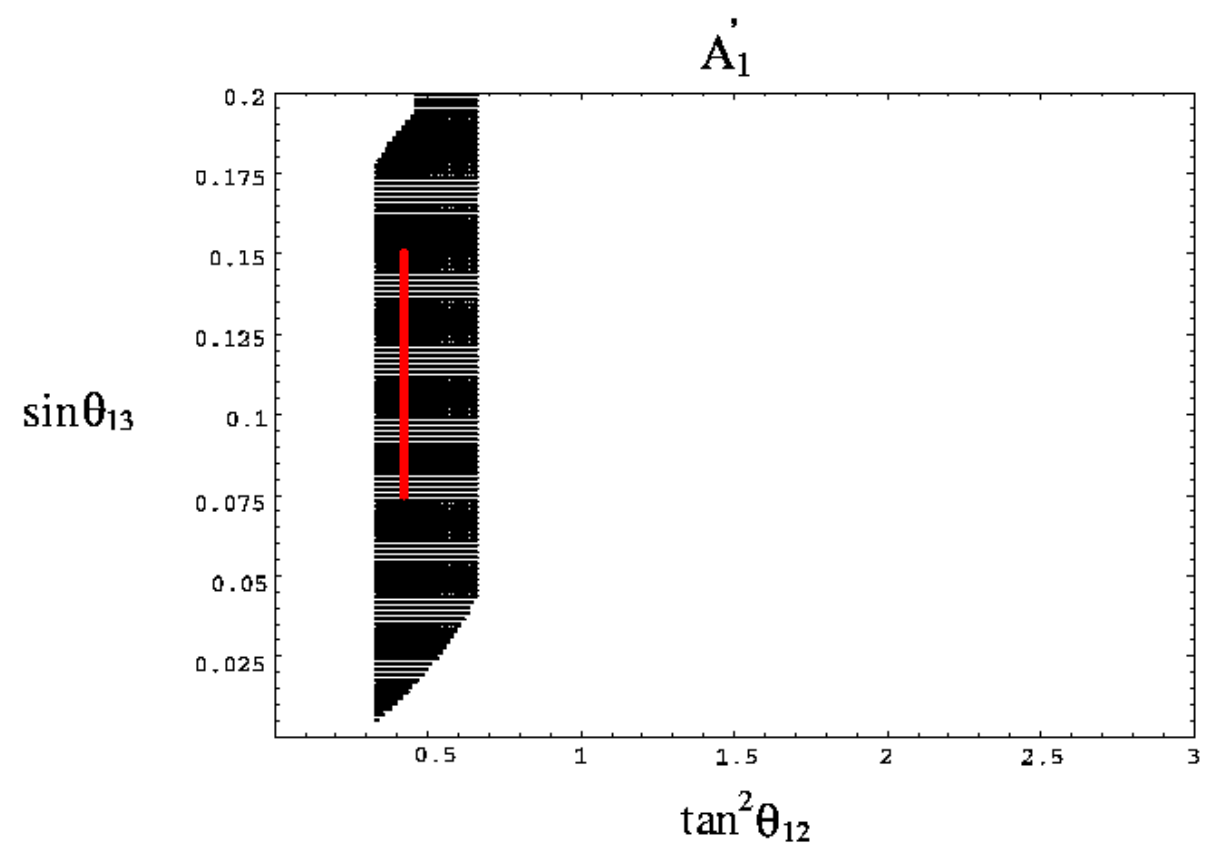

Figure 6: Scatter plot of $\sin \theta_{13}$ versus $\tan ^{2} \theta_{12}$ in the case of $\kappa=2 \bar{\omega}=0.07$ for $A_{1}^{\prime}$. The best fit is shown by a red line.

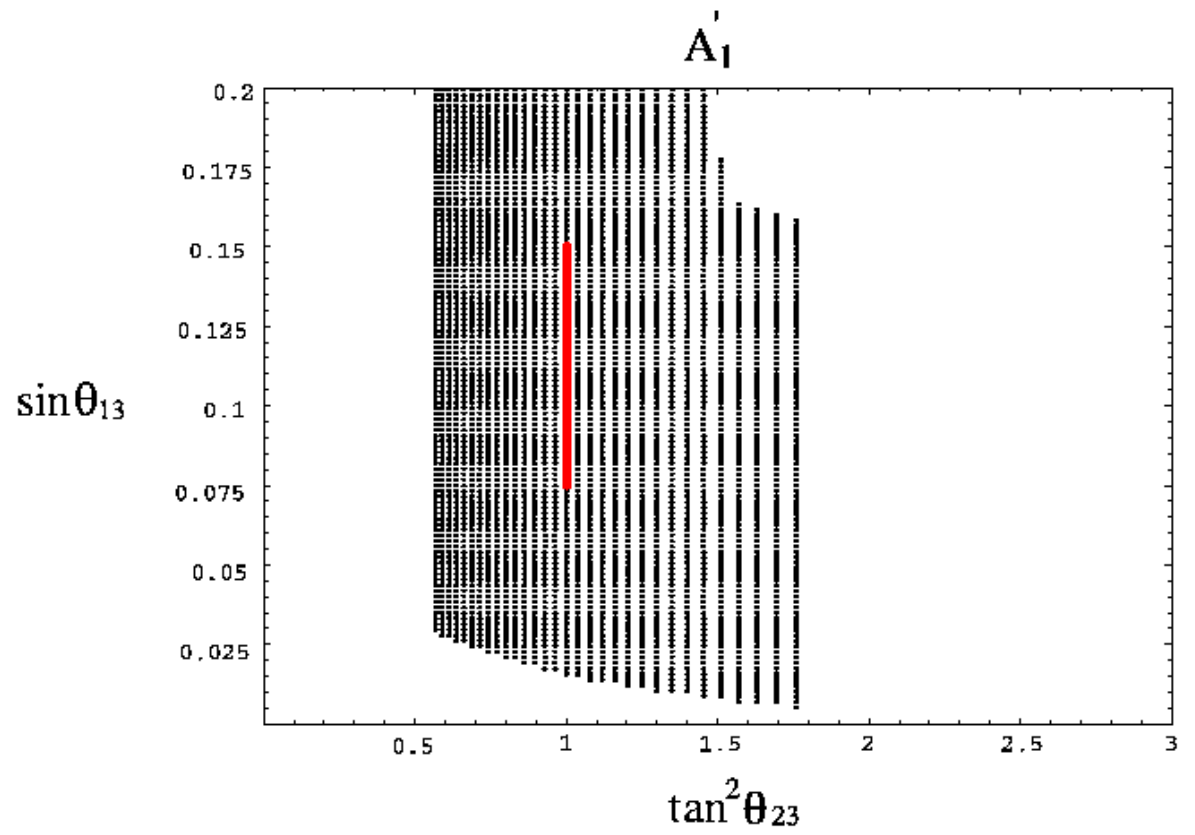

Figure 7: Scatter plot of $\sin \theta_{13}$ versus $\tan ^{2} \theta_{23}$ in the case of $\kappa=2 \bar{\omega}=0.07$ for $A_{1}^{\prime}$. The best fit is shown by a red line. 


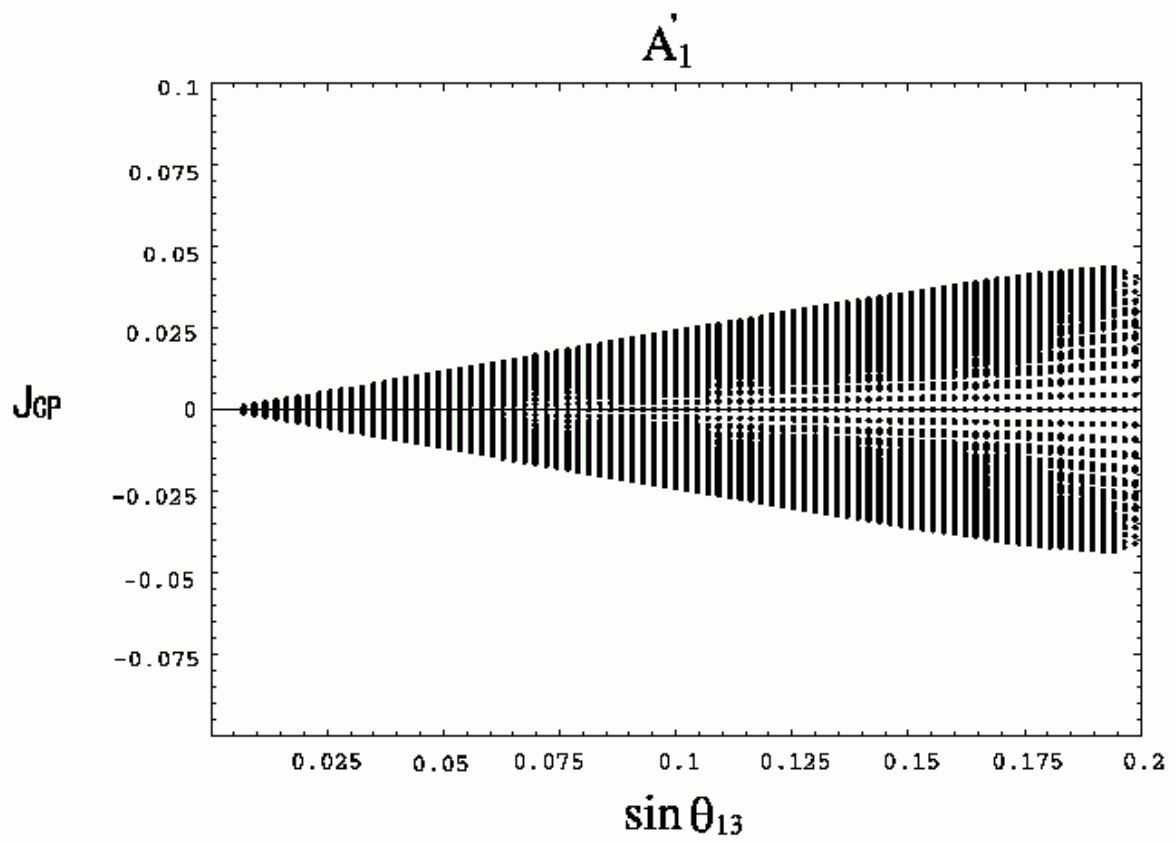

Figure 8: Scatter plot of $J_{C P}$ versus $\sin \theta_{13}$ in the case of $\kappa=2 \bar{\omega}=0.07$ for $A_{1}^{\prime}$.

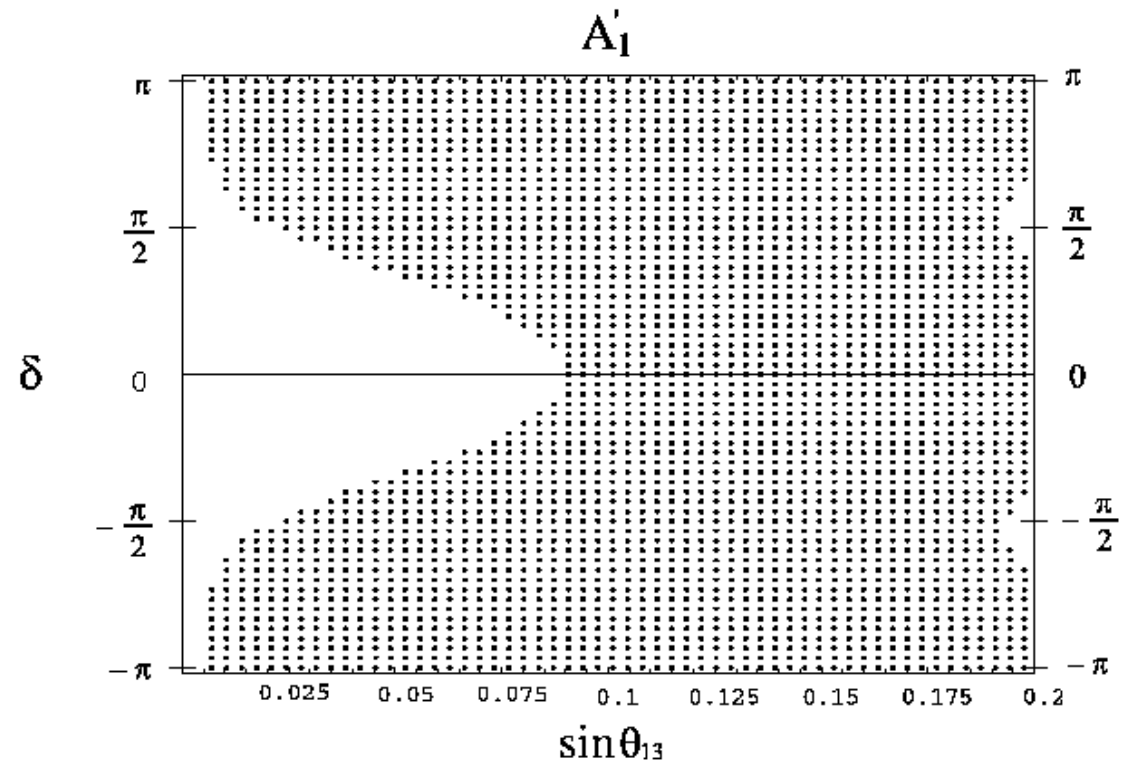

Figure 9: Scatter plot of $\delta$ versus $\sin \theta_{13}$ for $A_{1}^{\prime}$. 


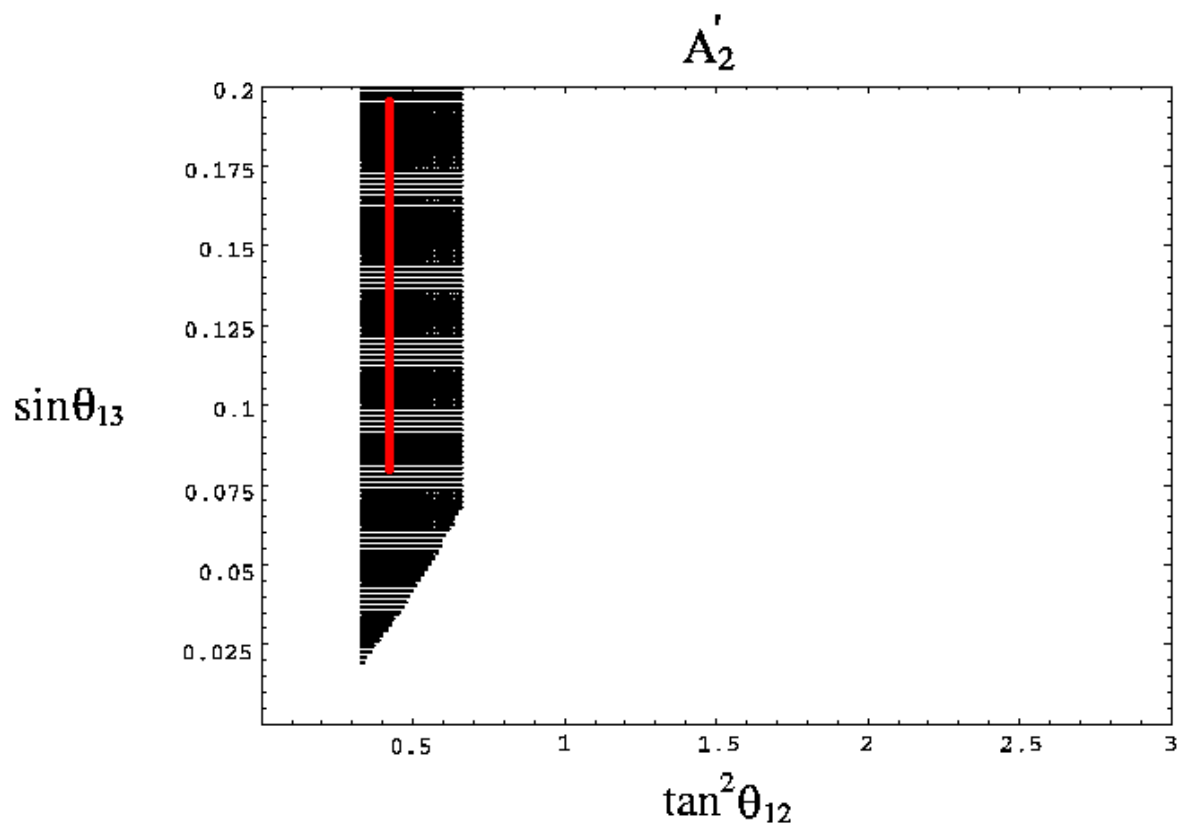

Figure 10: Scatter plot of $\sin \theta_{13}$ versus $\tan ^{2} \theta_{12}$ in the case of $\kappa=2 \bar{\omega}=0.07$ for $A_{2}^{\prime}$. The best fit is shown by a red line.

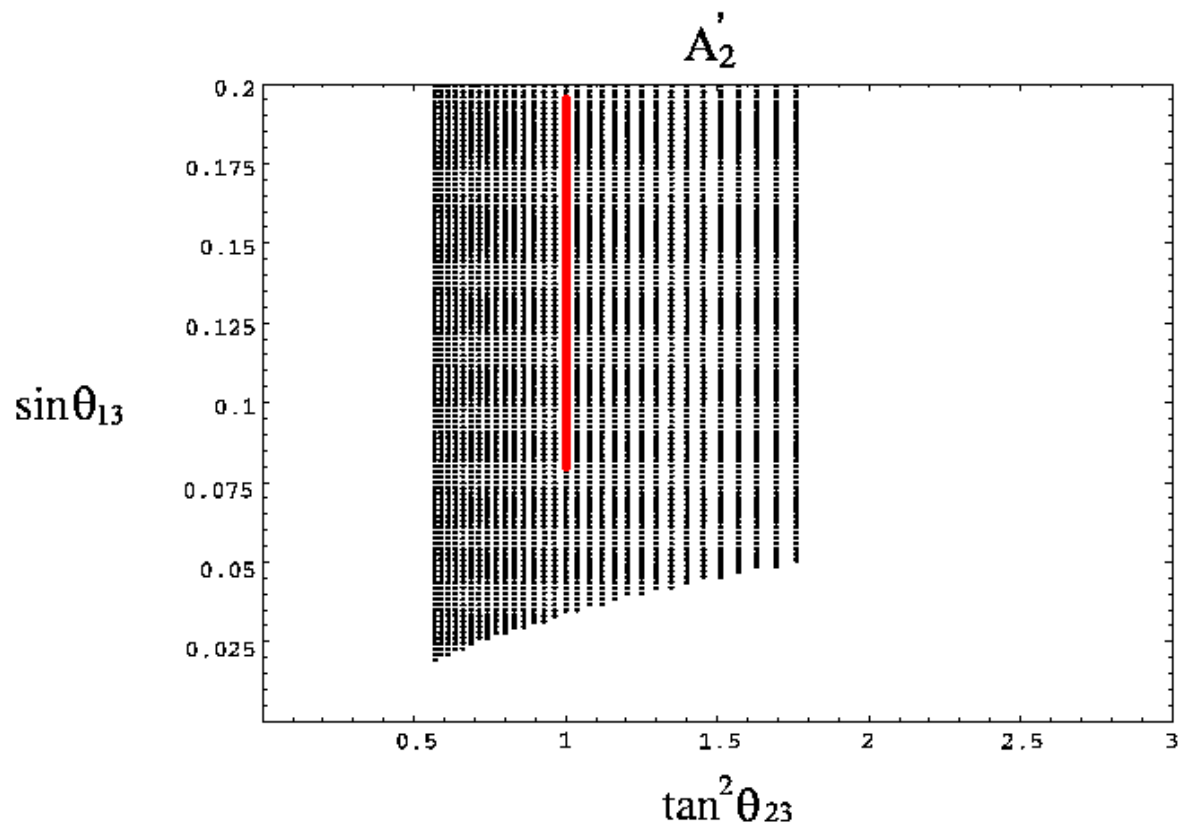

Figure 11: Scatter plot of $\sin \theta_{13}$ versus $\tan ^{2} \theta_{12}$ in the case of $\kappa=2 \bar{\omega}=0.07$ for $A_{2}^{\prime}$. The best fit is shown by a red line. 


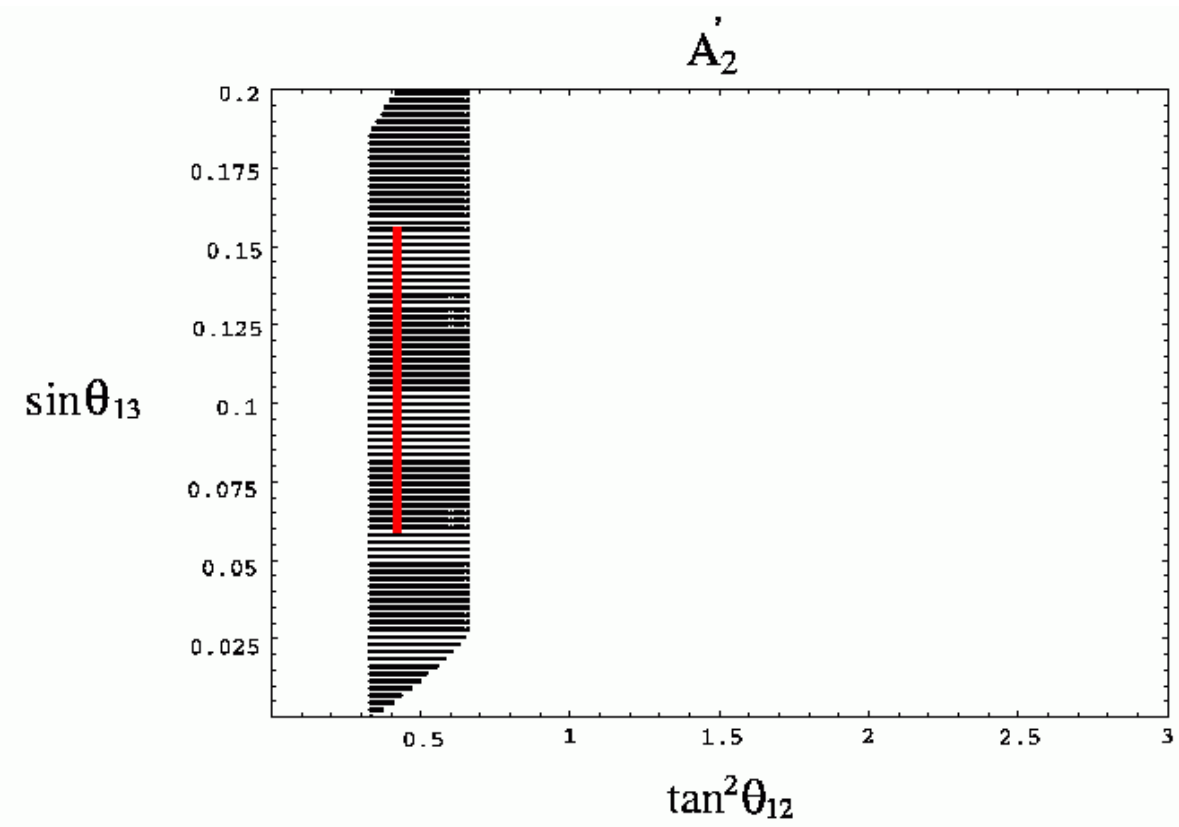

Figure 12: Scatter plot of $\sin \theta_{13}$ versus $\tan ^{2} \theta_{23}$ in the case of $\tan \theta_{12} \tan \theta_{23}<0$ with $\kappa=2 \bar{\omega}=0.07$ for $A_{2}^{\prime}$. The best fit is shown by a red line.

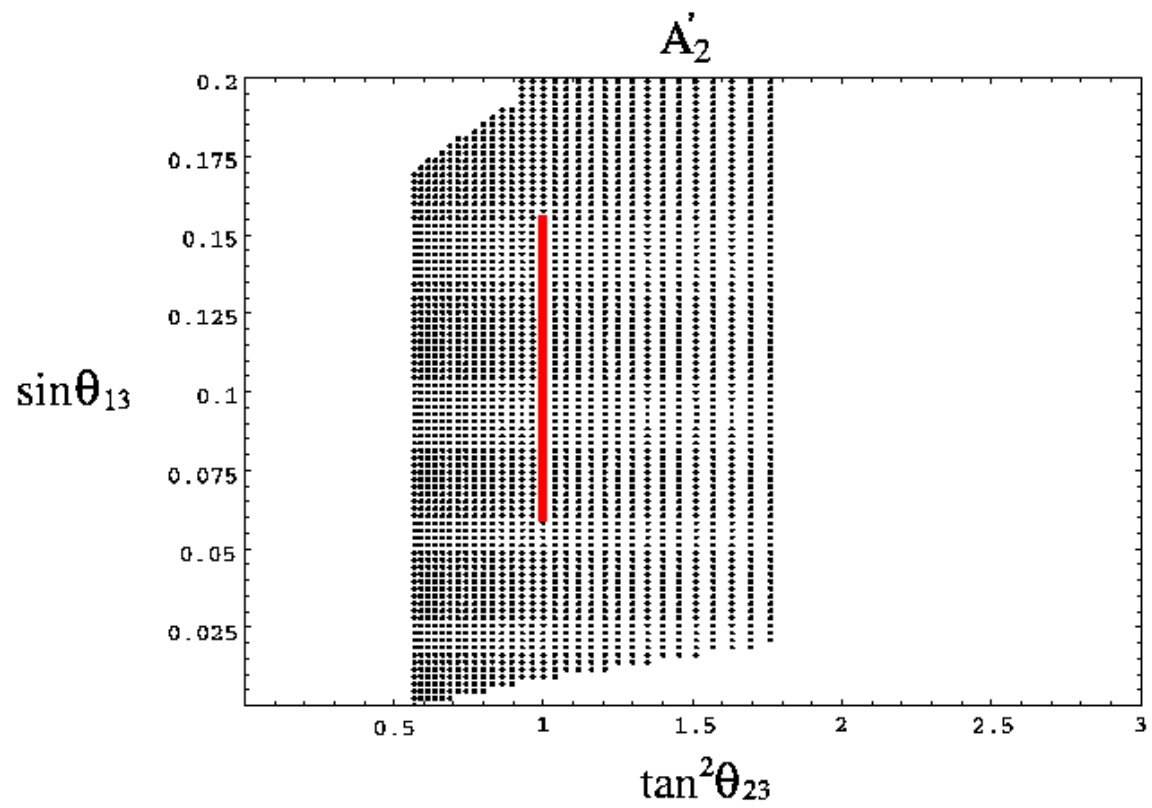

Figure 13: Scatter plot of $\sin \theta_{13}$ versus $\tan ^{2} \theta_{23}$ in the case of $\tan \theta_{12} \tan \theta_{23}<0$ with $\kappa=2 \bar{\omega}=0.07$ for $A_{2}^{\prime}$. The best fit is shown by a red line. 


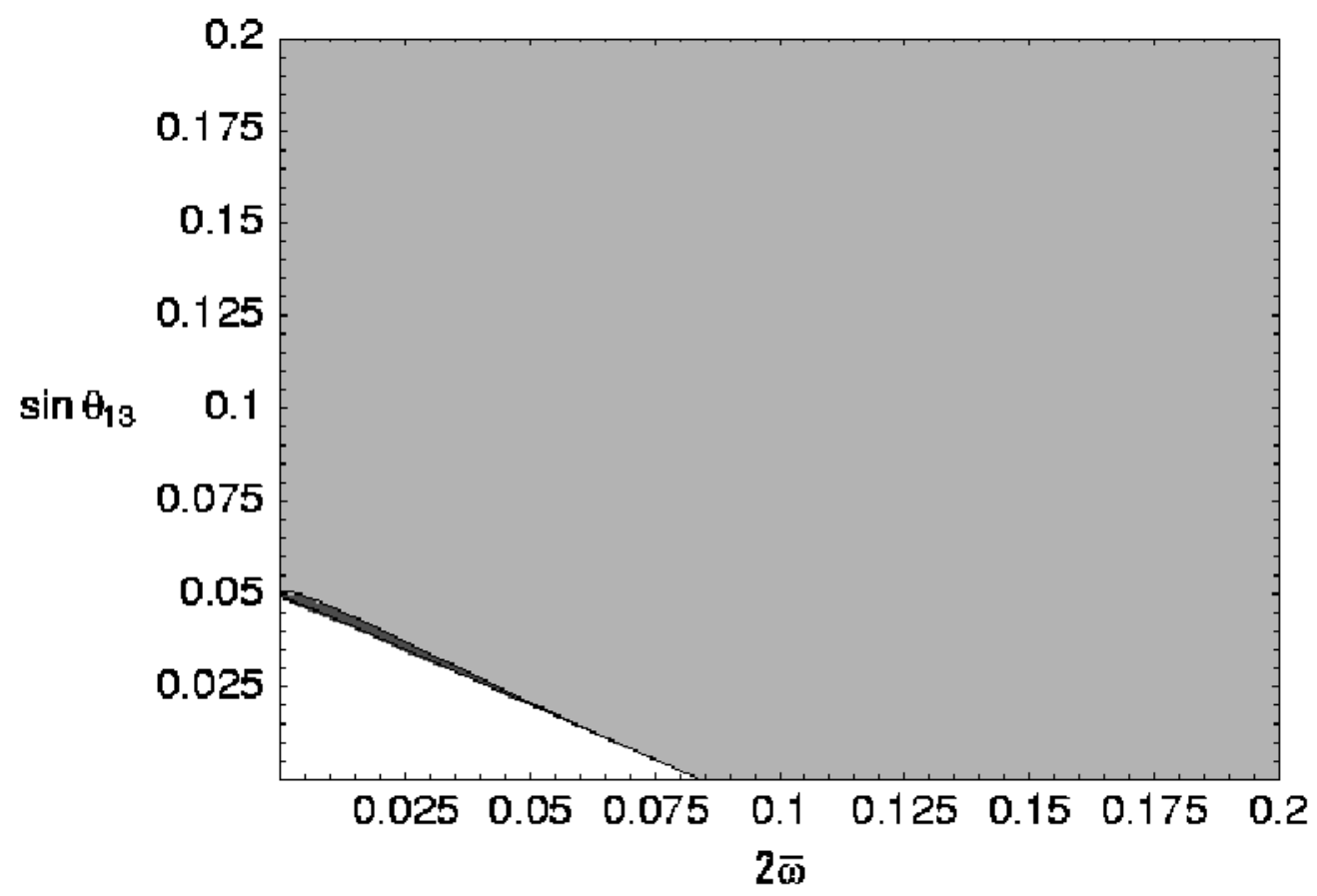

Figure 14: Prediction of $\sin \theta_{13}$ versus $2 \bar{\omega}$ in the case of $\bar{\epsilon}=0$. The gray region is allowed by the experimental data for $A_{1}^{\prime}$. The deep gray region is added for $A_{2}^{\prime}$.

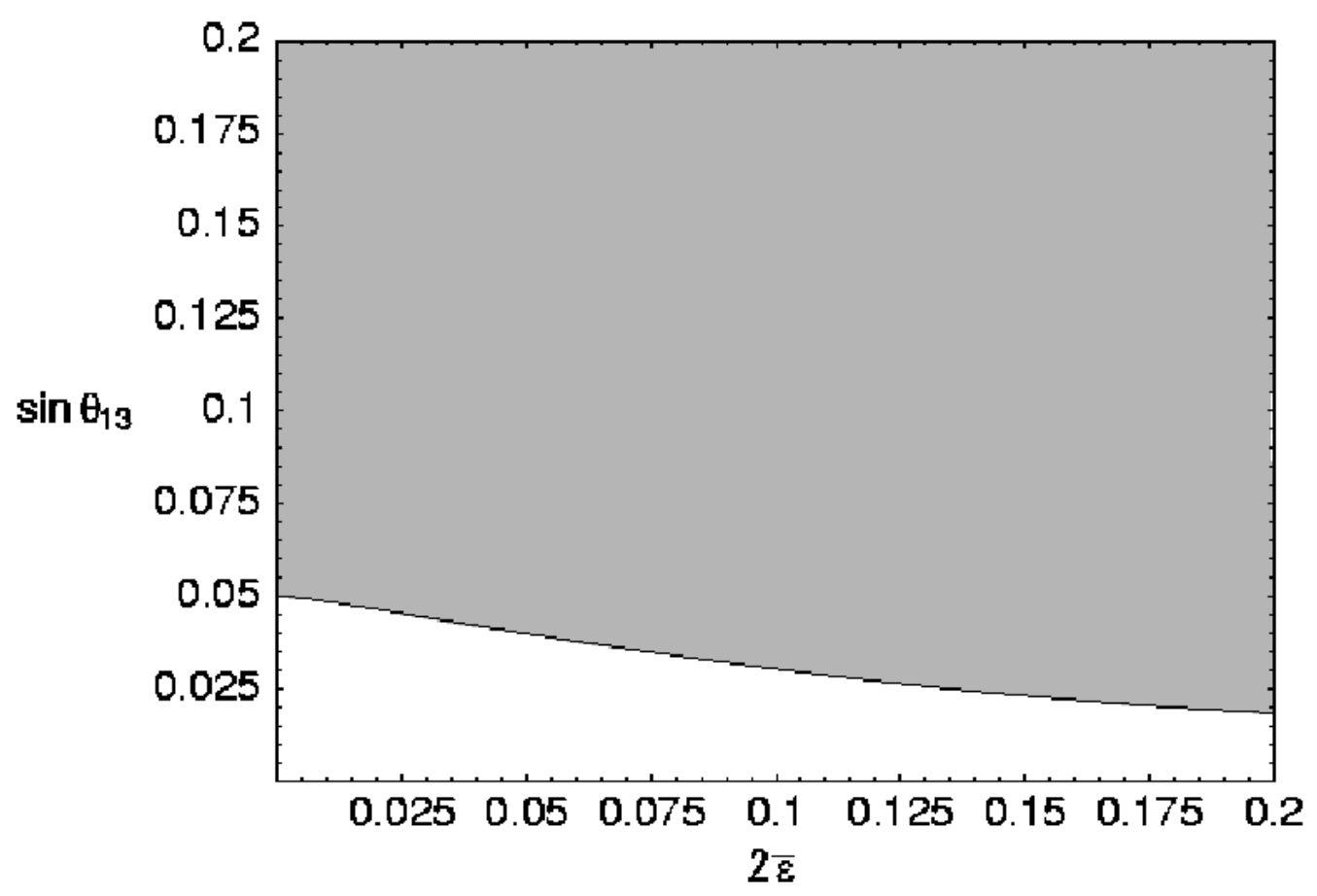

Figure 15: Prediction of $\sin \theta_{13}$ versus $2 \bar{\epsilon}$ in the case of $\bar{\omega}=0$ for both $A_{1}^{\prime}$ and $A_{2}^{\prime}$. The gray region is allowed by the experimental data. 